\title{
Médicaliser des urgences préhospitalières ... afin d'éviter l’hôpital!
}

\author{
A. Engeler ${ }^{a}$, D. Vadilonga ${ }^{a}$, \\ B. Savary-Borioli ${ }^{b}$, R. Mauria ${ }^{a, b}$
}

a Servizio di cardioanestesia e cure intense, Cardiocentro Ticino Lugano

b Croce Verde Lugano

1 Règlement interne de la «Croce Verde Lugano».

Correspondance:

Dr Romano Mauri

Président de la Croce Verde Lugano

Cardiocentro Ticino

Via Tesserete 48

CH-6900 Lugano

E-mail:

romano.mauri@cardiocentro.org

\section{Introduction}

Classiquement, l'évaluation de l'efficacité de la chaîne de sauvetage se base sur l'analyse des éléments chronologiques des différentes étapes de la prise en charge du patient, sur la qualité des soins prodigués par les services de secours ainsi que sur le devenir des malades en termes de morbidité et mortalité.

La médicalisation des urgences préhospitalières, à part offrir une compétence supplémentaire aux patients dont le pronostic vital est engagé, présente un bénéfice collatéral souvent ignoré mais néanmoins déterminant dans l'optique des services de santé publique: C'est l'hospitalisation évitée de par le fait que le médecin d'urgence sur place a pu exclure l'atteinte grave présumée qui avait initialement motivé son engagement.

\section{But de l'étude}

La présente étude a comme objectif d'analyser les missions préhospitalières qui grâce à l'intervention du médecin d'urgence ont permis soit d'établir le diagnostic définitif et de prodiguer au patient la totalité du traitement nécessaire directement sur place, soit d'orienter le malade vers une structure d'investigation et de traitement autre qu'un hôpital, par exemple en l'adressant à un cabinet médical.

\section{Patients et méthode}

Le service de secours «Croce Verde Lugano» (CVL) intervient sur un territoire de 84 communes du Sottoceneri ce qui correspond à une superficie de $320 \mathrm{~km}^{2}$ et un bassin de 131000 habitants équivalent à $40 \%$ de la population résidente du Canton du Tessin.

L'activité et les compétences des secouristes professionnels engagés auprès de la CVL sont très bien codifiées. Précisons qu'ils ne sont pas autorisés à laisser un patient à domicile sans avis médical, qu'il s'agisse d'un refus de soins exprimé par le malade ou de la présomption d'une affection bénigne [1].

La médicalisation du service de secours de la «Croce Verde Lugano» a débuté il y a quelques années déjà, mais c'est au cours de l'année 2003 qu'a été instauré une couverture plus systématique selon le principe du «rendez-vous». Cepen- dant, la structure actuelle ne permet pas encore d'assurer une présence sur le terrain de 24 h/24. Toutefois au vu du consensus établi entre les protagonistes des différents groupes professionnels, cet objectif devrait être atteint dans les meilleurs délais.

De façon rétrospective, ont été analysées les interventions médicalisées de l'année 2004, soit que le médecin d'urgence ait participé directement à l'intervention, soit qu'il ait aidé les secouristes professionnels à distance (télémédecine). Par la suite, le nombre et la proportion des patients non hospitalisés ont été comparés à ceux des deux années précédentes.

\section{Résultats}

Durant l'année 2004, les médecins d'urgence de la CVL ont effectué 873 missions dans le terrain, ce qui correspond à un taux de médicalisation de presque $17 \%$ de toutes les interventions primaires (873/5309). Dans 254 autres cas, leur contribution se limitait à une prise de décision à distance ( $5 \%$ de télémédecine). Donc, à peu près un patient sur quatre a bénéficié d'un avis médical lors de sa prise en charge en situation d'urgence préhospitalière.

Parmi toutes les interventions médicalisées, on retrouve 323 personnes avec un indice de gravité NACA 0-2 qui n'ont pas été hospitalisées (248 en 2002). Sur un total de 1794 malades avec un degré d'atteinte comparable pris en charge par la CVL, cela représente le $18 \%$. Cependant, les informations contenues dans la banque de données ne précisent pas quelle proportion des personnes non transportées a été adressée soit au médecin traitant ou au médecin de garde, soit laissée à domicile au terme des procédures diagnostiques et thérapeutiques (tab. 1).

La figure 1 illustre l'évolution de l'orientation des patients avec un indice NACA de 0 à 2 durant les années 2002-2004 moyennant une simple distinction entre le nombre de patients hospitalisés et ceux qui n'ont pas été transportés en ambulance. On observe une augmentation progressive du pourcentage des cas bénins qui évitent une consultation auprès d'un service d'urgence hospitalier (de 13 à 18\%). Au niveau statistique, cette progression constitue une diffé- 
Figure 1

Représentation graphique de l'orientation des patients NACA 0-2 (exprimée en \%) durant les années 2002-2004.

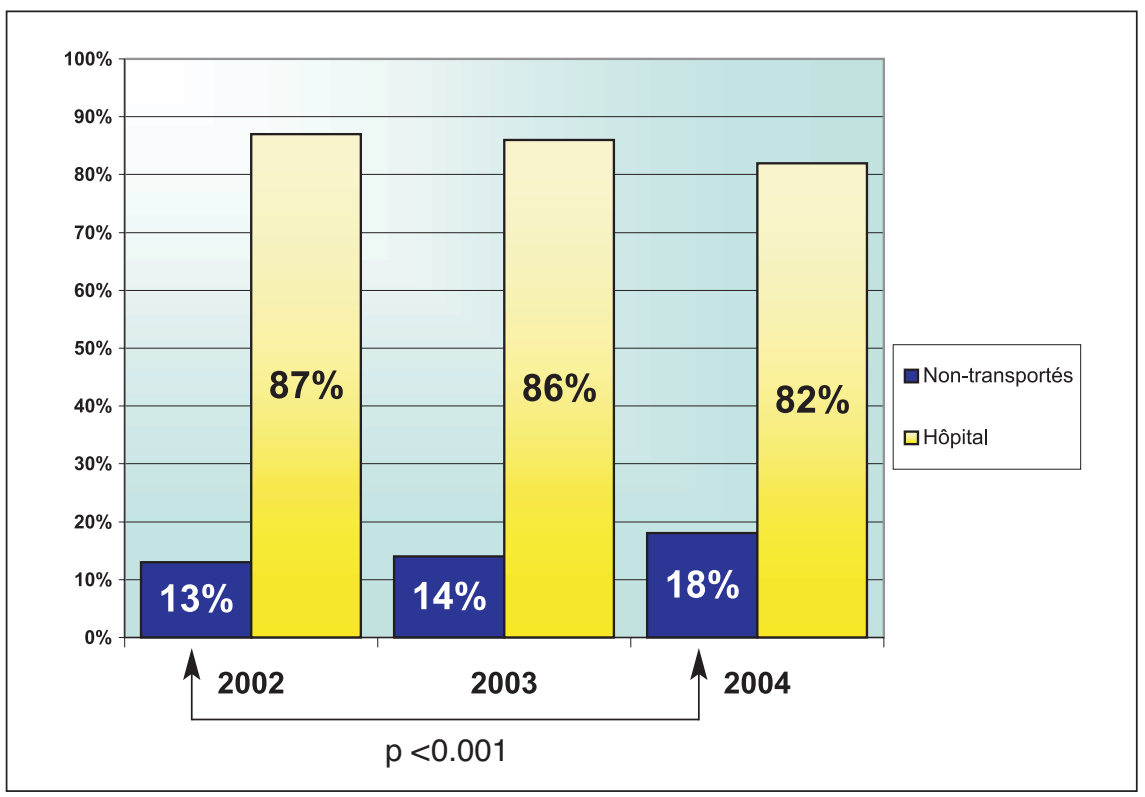

\section{Tableau 1}

Destination des patients NACA 0-2 durant les années 2002-2004.

\begin{tabular}{|lllr}
\hline Année & Hôpital & Non-transportés & Total \\
\hline 2002 & $1674(87 \%)$ & $248(13 \%)$ & 1922 \\
\hline 2003 & $1798(86 \%)$ & $293(14 \%)$ & 2091 \\
\hline 2004 & $1471(82 \%)$ & $323(18 \%)$ & 1794 \\
\hline
\end{tabular}

2 Hoby G. et al. Notfälle direkt ins Spital? Ein wichtiger Mechanismus der Kostenlawine. Schweiz Ärztezeitung 2005;86(27):1694-5. rence significative vu que la distribution entre les années 2002 et 2004 répond aux critères du test chi-carré avec une valeur de $\mathrm{p} \leq 0,001$.

\section{Discussion et conclusions}

Nous observons que grâce à un avis médical précoce (donné par un médecin d'urgence sur le site, soit par le biais de la télémédecine), à peu près une personne par jour en moyenne a pu éviter une consultation auprès d'un service d'urgence hospitalier dans la région de Lugano.

Bien que ce nombre soit encore faible, force est de constater qu'une tendance à une meilleure orientation des patients d'un degré de gravité se situant entre un indice NACA de 0 à 2 se dégage progressivement au cours des trois dernières années; et cette différence est significative.

La récente expérience de la «Croce Verde Lugano» permet de conclure que la médicalisation préhospitalière apporte une contribution déterminante à la prise en charge adéquate des malades.

Bien que la médecine d'urgence préhospitalière soit souvent considérée comme le bras prolongé de l'hôpital, il n'est nullement impératif que chaque patient pris en charge soit amené vers a un service d'accueil et d'urgence hospitalier pour y poursuivre les investigations ou le traitement. En termes de santé publique et en termes de coût de la santé, le bénéfice paraît évident [2].

Par conséquent, cet aspect de la médicalisation devrait être documenté de façon plus précise et mériterait une attention plus soutenue dans le cadre des discussions autour de l'efficacité de la chaîne de sauvetage. 